\title{
BMJ open Optimising drug prescribing and dispensing in subjects at risk for drug errors due to renal impairment: improving drug safety in primary healthcare by low eGFR alerts
}

\author{
Hanneke Joosten, ${ }^{1}$ lefke Drion, ${ }^{2}$ Kees J Boogerd, ${ }^{3}$ Emiel V van der Pijl, ${ }^{3}$ \\ Robbert J Slingerland, ${ }^{4}$ Joris P J Slaets, ${ }^{1,5}$ Tiele J Jansen, ${ }^{6}$ Olof Schwantje, ${ }^{7}$ \\ Reinold O B Gans, ${ }^{1}$ Henk J G Bilo ${ }^{2}$
}

To cite: Joosten $\mathrm{H}$, Drion I, Boogerd KJ, et al. Optimising drug prescribing and dispensing in subjects at risk for drug errors due to renal impairment: improving drug safety in primary healthcare by low eGFR alerts. BMJ Open 2013;3:e002068. doi:10.1136/bmjopen-2012002068

- Prepublication history and additional material for this paper are available online. To view these files please visit the journal online (http://dx.doi.org/10.1136/ bmjopen-2012-002068).

Received 9 September 2012 Revised 9 December 2012 Accepted 19 December 2012

This final article is available for use under the terms of the Creative Commons Attribution Non-Commercial 2.0 Licence; see http://bmjopen.bmj.com

For numbered affiliations see end of article.

Correspondence to Drs Hanneke Joosten; j.m.h.joosten@umcg.nl

\section{ABSTRACT}

Objectives: To assess the risk of medication errors in subjects with renal impairment (defined as an estimated glomerular filtration rate (eGFR) $\leq 40 \mathrm{ml} / \mathrm{min} /$ $1.73 \mathrm{~m}^{2}$ ) and the effectiveness of automatic eGFR $\leq 40$ alerts relayed to community pharmacists.

Design: Clinical survey.

Setting: The city of Zwolle, The Netherlands, in a primary care setting including 22 community pharmacists and 65 general practitioners.

Participants: All adults who underwent ambulatory creatine measurements which triggered an eGFR $\leq 40$-alert.

Primary and secondary outcome measures: The total number of ambulatory subjects with an eGFR $\leq 40$-alert during the study period of 1 year and the number of medication errors related to renal impairment. The type and number of proposed drug adjustments recommended by the community pharmacist and acceptance rate by the prescribing physicians. Classification of all medication errors on their potential to cause an adverse drug event (ADE) and the actual occurrence of ADEs (limited to those identified through hospital record reviews) 1 year after the introduction of the alerts.

Results: Creatine measurements were performed in 25929 adults. An eGFR $\leq 40$-alert was indicated for $5.3 \%(n=1369)$. This group had a median (IQR) age of $78(69,84)$ years, and in $73 \%$ polypharmacy $(\geq 5$ drugs) was present. In $15 \%$ ( $n=211)$ of these subjects, a medication error was detected. The proportion of errors increased with age. Pharmacists recommended 342 medication adjustments, mainly concerning diuretics $(22 \%)$ and antibiotics (21\%). The physicians' acceptance rate was $66 \%$. Of all the medication errors, $88 \%$ were regarded as potential ADEs, with most classified as significant or serious. At follow-up, the ADE risk $(n=40)$ appeared highest when the proposed medication adjustments were not implemented (38\% vs $6 \%)$.

Conclusions: The introduction of automatic eGFRalerts identified a considerable number of subjects who

\section{ARTICLE SUMMARY}

Article focus

- To evaluate the number of subjects with at risk for medication errors due to renal impairment (defined as estimated glomerular filtration rate (eGFR) $\leq 40 \mathrm{ml} / \mathrm{min} / \mathrm{m}^{2}$ ) in a primary care setting.

- To assess the risk of medication errors in subjects with renal impairment.

- To evaluate the effectiveness of generating automatic eGFR $\leq 40$-alerts and medication reviews involving community pharmacists.

Key messages

- Providing renal laboratory data to pharmacists in a primary care setting revealed that there were a considerable number of subjects at increased risk for adverse drug events (ADEs) due to renal impairment.

- The issuance of eGFR alerts allowed community pharmacists to provide valuable medication adjustment recommendations to the prescribing physicians, with a good acceptance rate.

- The implementation of this simple protocol could identify many potential ADEs, thereby substantially reducing the risks of unnecessary iatrogenic damage in subjects with impaired renal function.

Strengths and limitations of this study

- Implementation of this protocol in clinical practice is possible in various healthcare settings.

- Increased collaboration with community pharmacists improved healthcare safety and awareness of medication errors related to renal function impairment in primary care.

- Extending the availability of laboratory renal data which were not formerly shared is relatively straightforward with minimal expense.

- Effect of eGFR alerts on the incidence of ADEs could not be measured.

- Study design does not allow either the determination of individual healthcare effects or an overall cost-benefit analysis of this healthcare safety strategy. 
are at risk for ADEs due to renal impairment in an ambulatory setting. The nationwide implementation of this simple protocol could identify many potential ADEs, thereby substantially reducing iatrogenic complications in subjects with impaired renal function.

\section{INTRODUCTION}

Safe medication management is an important healthcare topic, as medication errors are a significant source of iatrogenic injury to patients. ${ }^{1-7}$ Injuries resulting from such errors are known as adverse drug events (ADEs). Various factors are associated with ADEs, including patient characteristics, lack of medication monitoring and prescription errors. ${ }^{4-6} 8$ Studies on medicationrelated hospital admissions estimate that $21-91 \%$ of

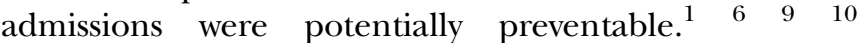
Important patient determinants for ADEs are increasing age, female gender, polypharmacy, non-compliance and comorbidities such as cognitive dysfunction or renal impairment. $^{1-4} 7810$

Renal impairment is a well-known risk factor for ADEs, but it often remains unrecognised by physicians and pharmacists. ${ }^{1-14}$ Even in high-risk patients such as the elderly and those with diabetics, healthcare workers are not always sufficiently alert. ${ }^{15-17}$ Various studies reported considerable dosing difficulties and subsequent medication errors in patients with renal impairment. $^{10} \quad 12$ 17-19 Therefore, intensified collaboration between healthcare workers (such as general practitioners (GPs), pharmacists and nephrologists) is recommended with exchange of relevant patient information (medical history and comorbidities) and more effective use of routinely collected data from electronic patient records such as laboratory results relating to renal function. 26 20-23

In this 1-year observational study, we aimed to evaluate the number of subjects at risk for medication errors due to renal impairment (defined as an estimated glomerular filtration rate $(\mathrm{eGFR}) \leq 40 \mathrm{ml} / \mathrm{min} / 1.73 \mathrm{~m}^{2}$ ) and the effectiveness of providing automatically generated eGFR $\leq 40$-alerts towards community pharmacists in a shared pharmaceutical care model. In addition, we classified all medication errors for their potential to cause ADEs and evaluated the actual number of ADEs in those with a medication error after a period of 1 year.

\section{MATERIALS AND METHODS \\ Setting}

This study was conducted in Zwolle, which is a city in the north of the Netherlands with a population of more than 89000 adults. $^{24}$ All of the primary care pharmacies $(n=11)$ and the general practices $(n=24)$ participated in this study. Their characteristics are shown in table 1. Dutch patients are generally registered at one single pharmacy and GP practice, which promotes continuity of care and reliable information regarding each individual's medication use. Secondary care in this region is delivered by the Isala Clinics, a 1000+bed teaching hospital in Zwolle. All standard laboratory investigations requested in both primary and secondary care are

Table 1 Characteristics of participating pharmacists and GPs and their practices

\begin{tabular}{|c|c|c|}
\hline Characteristics & Pharmacists & GPs \\
\hline \multicolumn{3}{|l|}{ Participants } \\
\hline Number (\%) & $22(100)$ & $65(100)$ \\
\hline \multicolumn{3}{|l|}{ Sex, $n(\%)$} \\
\hline Male & $9(40)$ & $42(65)$ \\
\hline Female & $13(60)$ & $23(35)$ \\
\hline \multicolumn{3}{|l|}{ Years in practice, $\mathrm{n}(\%)$} \\
\hline $0-10$ & $10(45)$ & $25(39)$ \\
\hline $11-20$ & $9(41)$ & $15(23)$ \\
\hline $21-30$ & $0(0)$ & $21(32)$ \\
\hline$>30$ & $3(14)$ & $4(6)$ \\
\hline \multicolumn{3}{|l|}{ Position in practice, $\mathrm{n}(\%)$} \\
\hline (Joint) owner & $6(27)$ & $45(70)$ \\
\hline Employee & $16(73)$ & $20(30)$ \\
\hline \multicolumn{3}{|l|}{ Practice } \\
\hline Number (\%) & $11(100)$ & $24(100)$ \\
\hline \multicolumn{3}{|l|}{ Practice type, n (\%) } \\
\hline Independent & $9(80)$ & - \\
\hline Chain & $2(20)$ & - \\
\hline Overall number of patients, $n$ & 114.033 & 117.147 \\
\hline Practice size, median (IQR) & $10000(7000,14000)$ & $3426(2691,6586)$ \\
\hline \multicolumn{3}{|l|}{ Prescription system, $n(\%)$} \\
\hline Computer based & $11(100)$ & $24(100)$ \\
\hline
\end{tabular}


performed in one laboratory, which uses a single electronic system for data handling.

\section{Design and case finding}

This prospective observational study was conducted between 1 February 2009 and 31 January 2010. During this period, all consecutive adults in whom a serum creatine was measured in the ambulatory setting and who had an eGFR at or below the cut-off point of $40 \mathrm{ml} /$ $\mathrm{min} / 1.73 \mathrm{~m}^{2}$ were identified, irrespective of the reason for laboratory testing. This threshold was based on guidelines advising dosage adjustment in renal impairment ${ }^{2526}$ and also chosen from a practical point of view. A higher cut-off point of $50-60 \mathrm{ml} / \mathrm{min} / 1.73 \mathrm{~m}^{2}$ was expected to exceed an acceptable workload, and the generation of many alarms induces the risk of ignoring and overriding alerts. Each week, the laboratory automatically generated a report for any ambulatory patients with an eGFR $\leq 40 \mathrm{ml} / \mathrm{min} / 1.73 \mathrm{~m}^{2}$ for the pharmacists.

\section{Study protocol}

A predefined protocol was followed after the pharmacist received a report on an eGFR $\leq 40 \mathrm{ml} / \mathrm{min} / 1.73 \mathrm{~m}^{2}$ (figure 1). First, the patients' pharmacist checked the actual medication regimen for current errors related to renal impairment. The numbers and types of errors were registered. Medication errors were based on Dutch Pharmacists' guidelines including 'the National Formulary on drug prescribing in renal impairment' and the 'National Shared Care Guidelines on Chronic Kidney Disease (CKD), ${ }^{25}{ }^{26}$ Second, the pharmacist alerted the prescribing physician (GP or clinician) on the low eGFR and, if appropriate, an adjusted medication regimen was recommended. Pharmacists contacted prescribing physicians by telephone or (if unreachable) by email. Finally, an alert warning for a low eGFR (eGFR $\leq 40$-alert) was activated in the patient's pharmacy record. This eGFR $\leq 40$-alert then appeared with every future new prescription. After this first laboratory notification, follow-up eGFR results were also reported to the pharmacists. When an eGFR recovered well beyond the cut-off value during follow-up (specified as an eGFR $>50 \mathrm{ml} / \mathrm{min} / 1.73 \mathrm{~m}^{2}$ ), the eGFR $\leq 40$-alert was removed from the pharmacy record.

The study was approved by the local medical ethics committee and conducted in accordance with the guidelines of the Declaration of Helsinki. All pharmacists and GPs informed their patients about the study through flyers, issued both at the pharmacies and at the GP practices. The patient folder and the Isala Clinics website

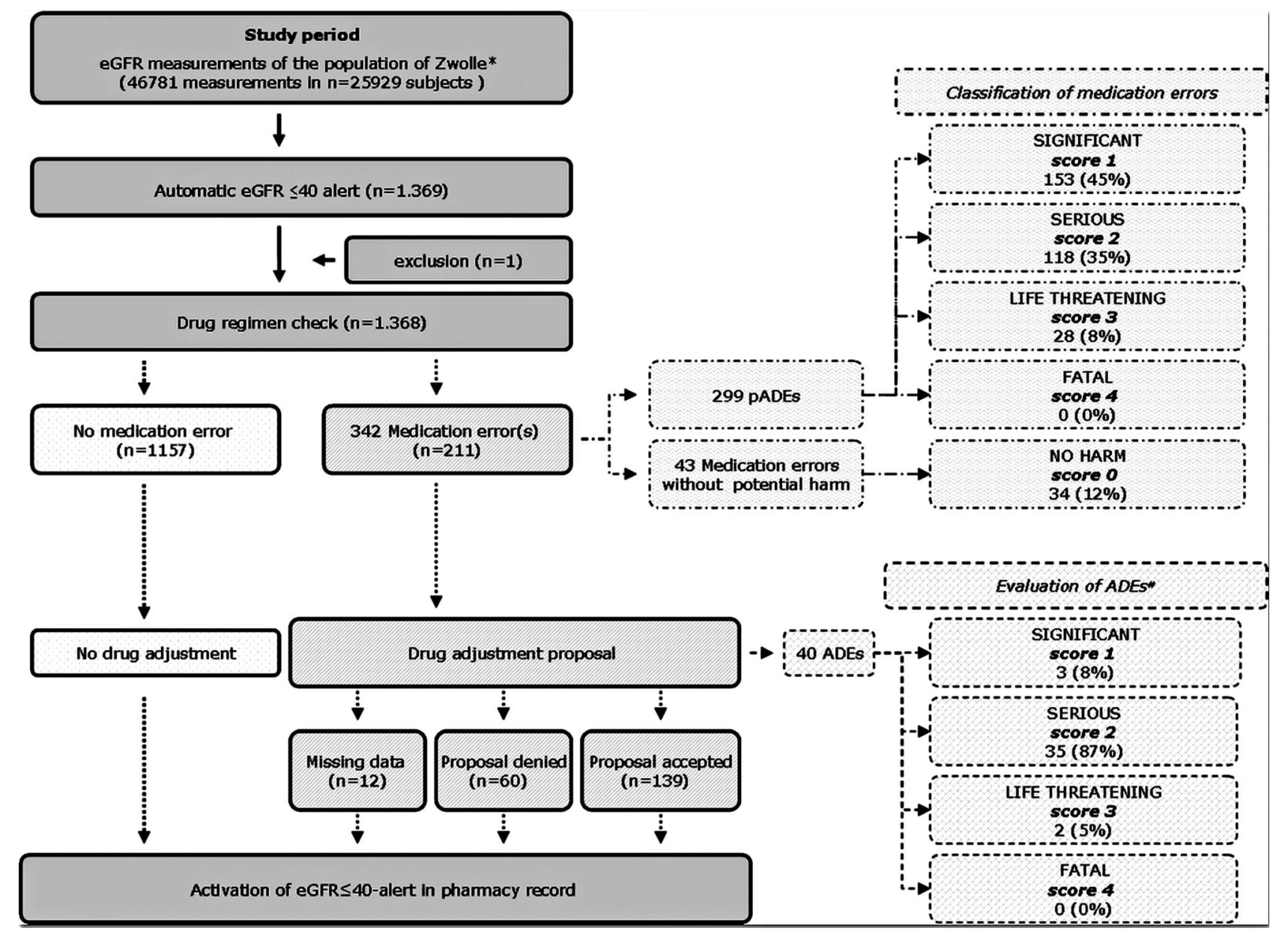

Figure 1 Flow chart summarising study method and selection of study population. 
also contained information about the stepwise eGFR $\leq 40$-alert protocol, the sharing of laboratory data and medication monitoring. The study had an opt-out policy; therefore, subjects who did not wish to participate in this pharmacovigilance study were excluded from the weekly reporting. It should be emphasised that the final decision about making any medication changes after an alert (and informing the patient) was considered to be the responsibility of the prescribing physician.

\section{Definitions and calculations}

Serum creatine was measured with an enzymatic essay (Modular, Roche, Mannheim, Germany), and eGFR was calculated with the enzymatic MDRD formula. ${ }^{27}$ The only medications included were those prescribed by healthcare professionals, and topical or over-the-counter products were excluded. Actual medication use was assessed by documenting all current prescriptions according to the Anatomical Therapeutic Chemical (ATC) classification system ${ }^{28}$ at the moment of the first eGFR $\leq 40$-alert. Polypharmacy was defined as the chronic ( $>1$ year) use of $\geq 5$ drugs.

\section{Data collection}

For all identified subjects with an eGFR $\leq 40$-alert, demographics and medication information were collected. Any medication adjustment recommendations were recorded, which included the patient's medical record number, the pharmacist, the type and daily dose of the medication, and the prescribing physician (GP or clinician). The physician's response to the pharmacist's recommendation was also recorded. Finally, the amount of time the pharmacists spent on every eGFR $\leq 40$-alert was documented.

\section{Classification and tracking of (potential) ADEs}

To evaluate the impact of eGFR $\leq 40$-alerts, two pharmacists (EVvdP and $\mathrm{KJB}$ ) independently evaluated all medication errors on the potential to cause an ADE (defined as a potential $\mathrm{ADE}(\mathrm{pADE}))$. They received a database that was anonymised by an investigator not involved in the eGFR-alert processing $(\mathrm{HJ})$. A methodology was developed for classification of medication errors and (p)ADEs. ${ }^{29}$ They judged and classified the theoretical severity of the medication error, yielding a score of $0-4$ $(0=$ drug error without significant harm, 1=potentially significant, $2=$ potentially serious, $3=$ potentially life threatening, 4=potentially fatal) (table 2). To reach a consensus, all discrepant ratings were discussed with both pharmacists and two nephrologists (HJGB and HJ). Examples of pADE classifications are listed in table 2. The best assessment of the number of ADEs proved to be from the documentation on ADEs in the hospital records. ${ }^{30}$ Therefore, 1 year after the end of the study, the hospital records of all subjects in whom a medication error was detected were reviewed. This review was performed by two nephrologists (HJ and HJGB) who independently checked the occurrence of ADEs. ADEs were based on admission and discharge diagnosis in the patients' medical records. The relationship of the ADE with the 'suspected' agent was double-checked by evaluating whether the medication regimen at admission in the hospital record matched with the pharmacy record at the date of admission. After a review of the hospital records, $\mathrm{HJ}$ and HJGB discussed their findings for reaching consensus.

\section{Data analysis}

The main outcome measures were the incidence of eGFR $\leq 40$-alerts, the number and types of medication

\begin{tabular}{lll} 
Table 2 & Categories of potential adverse drug events according to severity \\
\hline Score & Potential severity & Examples \\
\hline 0 & $\begin{array}{l}\text { Drug error without potential } \\
\text { harm }\end{array}$ & Not applicable \\
& Significant & Gastrointestinal complaints \\
& & Therapeutically ineffective dose according to eGFR \\
& Mild neurological effects (e.g. motoric dysfunction) \\
& Hepatic dysfunction \\
& Any significant event identified by patient which does not require change in \\
& therapy \\
& Sypoglycaemia \\
& Nephrotoxicity or increased risk nephrolithiasis \\
& Electrolyte disturbances (e.g. hyperpotassiemia) \\
& Altered mental status due to sedation \\
& Myopathy or rhabdomyolysis \\
& Gastrointestinal bleed \\
& Lactic acidosis \\
& Cardiac arrhythmia \\
& Life threatening & Decline in mental status with risk of falling \\
& Respiratory failure requiring intubation (e.g. bronchospasms) \\
& Death \\
\hline
\end{tabular}


errors and the number and types of medication adjustment proposals. Secondary outcome measures were the time required for pharmacists to process the eGFR $\leq 40$-alerts, the adherence of physicians to the proposed adjustments, risk factors for medication errors and the severity of medication errors. In addition, after 1 year of follow-up, we checked the incidence of ADEs in subjects in whom a medication error was detected. Statistical analysis was performed with SPSS V.16.0 (SPSS Inc., Chicago, Illinois, USA). Data are presented as the mean and SD when normally distributed. Otherwise, the median and (IQR) were used. For normally distributed data, the differences in baseline characteristics were evaluated with the independent samples $\mathrm{t}$ test. For nonparametric data, the Mann-Whitney $\mathrm{U}$ test was used. Differences in distribution were calculated using the $\chi^{2}$ tests.

\section{RESULTS \\ Incidence of eGFR $\leq \mathbf{4 0}$-alert and characteristics of the study population}

During the study period, 46781 creatine measurements were performed in 25929 subjects. In 5.3\% ( $\mathrm{n}=1369)$ of cases, an eGFR $\leq 40$-alert was indicated. One patient indicated no willingness to participate for privacy reasons, leaving 1368 subjects for analysis (figure 1). Their characteristics are summarised in table 3 . Overall, $56 \%$ were women, the median age was $78(69,84)$ years (distribution is shown in figure 2) and the median eGFR was 34 (27, 38) $\mathrm{ml} / \mathrm{min} / 1.73 \mathrm{~m}^{2}$. Overall, polypharmacy was present in $73 \%$ ( $\mathrm{n}=993)$ with a mean number of medications per patient of 7 (range 0-21). An overview of the actual medication use in the study population (which reflects comorbidities) according to the ATC classification is given in online supplementary appendix A.

\section{Number and type of medication errors}

Overall, 342 medication errors were detected in 211 patients with an eGFR $\leq 40$-alert ( $15 \%$ of the study population) (figure 1). The proportion of errors increased

\begin{tabular}{|c|c|}
\hline Variable & \\
\hline Number of subjects, n (\%) & $1368(100)$ \\
\hline \multicolumn{2}{|l|}{ Demographics } \\
\hline Age (years), median (IQR) & $78(69,84)$ \\
\hline Male, n (\%) & $601(44)$ \\
\hline Diabetes, n (\%) & $346(25)$ \\
\hline \multicolumn{2}{|l|}{ Renal variables } \\
\hline eGFR $\left(\mathrm{ml} / \mathrm{min} / 1.73 \mathrm{~m}^{2}\right)$, median (IQR) & $34(27,38)$ \\
\hline $\begin{array}{l}\text { Serum creatine }(\mu \mathrm{mol} / \mathrm{ml}) \text {, median } \\
(\mathrm{IQR})\end{array}$ & $152(128,186)$ \\
\hline \multicolumn{2}{|l|}{ Actual drug regimen } \\
\hline Number of drugs, median (IQR) & $7(4,9)$ \\
\hline Polypharmacy, n (\%) & $993(73)$ \\
\hline
\end{tabular}

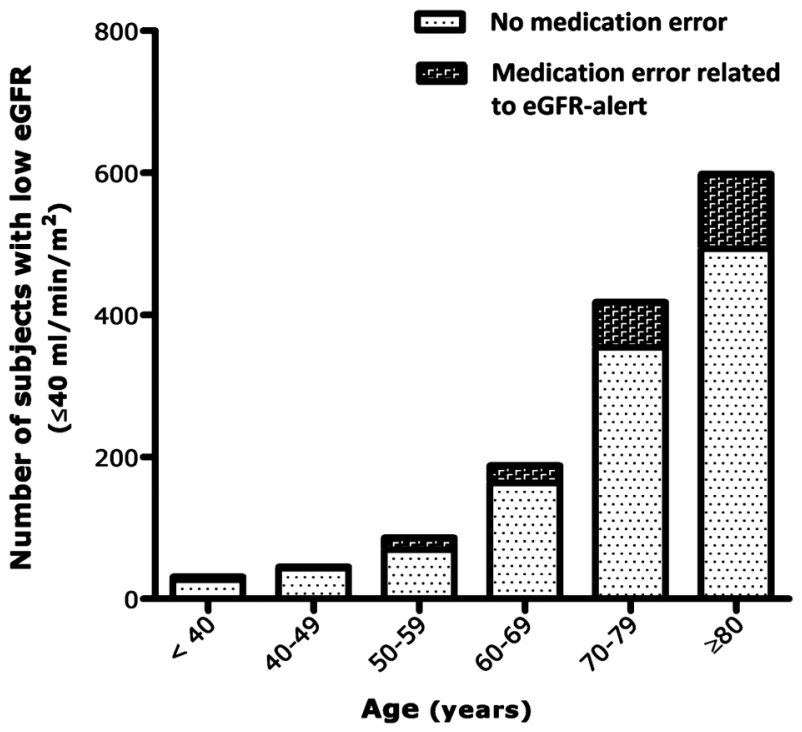

Figure 2 Age distribution of study population and risk of medication error per age category.

with increasing age (figure 2). The types of medication most commonly associated with errors were diuretics $(22 \%)$, antibiotics $(21 \%)$ and antigout medications (15\%) (figure 3). The majority of these medications (77\%) were prescribed by GPs. An overview of the type of medication errors that were identified by the pharmacists is given in figure 4 .

\section{Physicians' compliance with medication adjustment recommendations}

Figure 5 gives an overview of the frequency and types of medication adjustment recommendations. The most common recommendations were 'change dosage' $(55 \%)$, followed by 'stop medication' (24\%). In $31 \%$ $(\mathrm{n}=105)$, the proposal concerned a new prescription. Physicians complied with the recommendation in $66 \%$ $(n=226)$ of cases. In $28 \%(n=96)$ of cases, the pharmacists' advice was rejected and the medication regimen remained unchanged. The main reasons for rejection included already increased alertness with intensive monitoring by the prescribing physician (often being an internist or nephrologist) and an inadequate response to lower dosages in the past. The majority of rejected recommendations included diuretics and renin-angiotensin-aldosterone system blockers like ACE inhibitors and ARB drugs. In some cases, the recovery of renal function was expected or underestimation of renal function was presumed, both of which were generally checked with a $24 \mathrm{~h}$ creatine clearance. Overall, acutely reduced eGFR did not account for an important subset of the eGFR $<40$-alerts towards the community pharmacists $(n=3)$. Notably, in 22 of the 96 cases, the medication was soon changed anyway, due to a further decrease in the eGFR or the occurrence of an ADE. Therefore, from the latter it seems plausible that with the eGFR $\leq 40$-alert, the physician's awareness of the risk for an 
Figure 3 Medication groups associated with medication errors related to renal impairment.

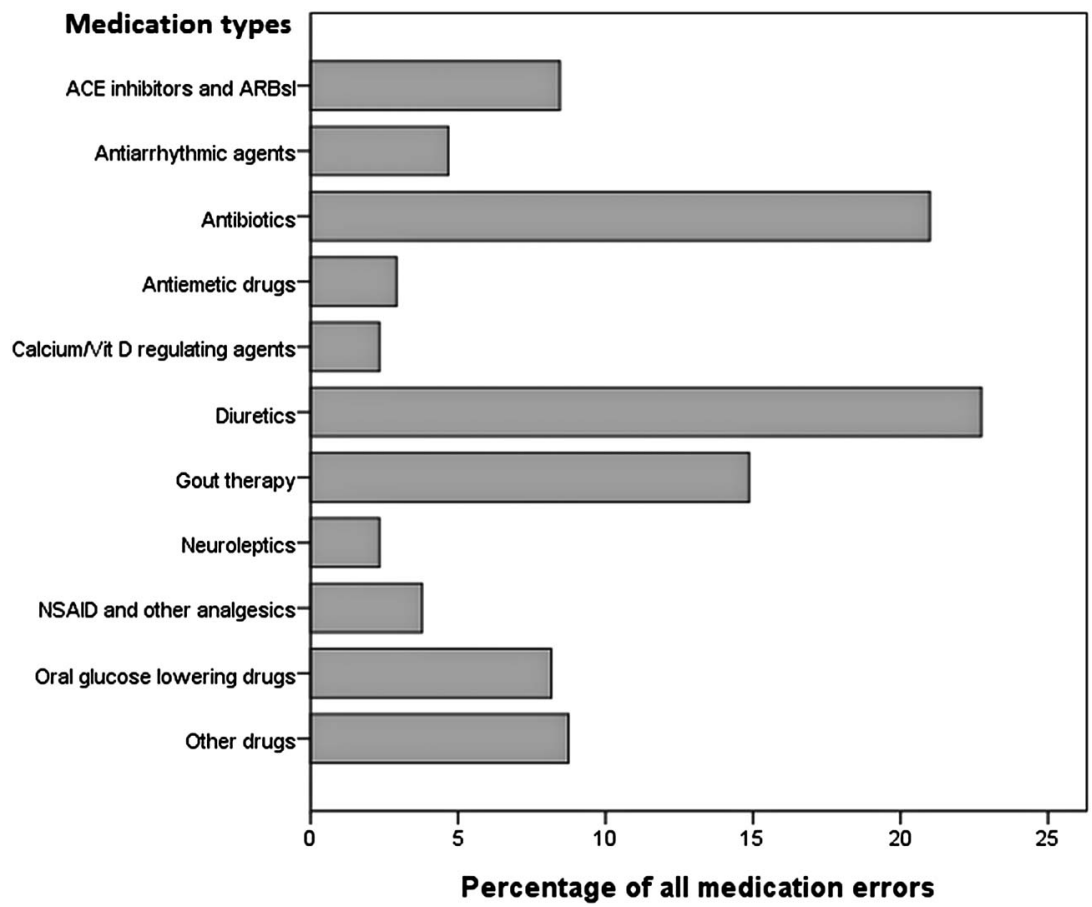

ADE was triggered. Data on rejection or agreement lacked in $6 \%(n=20)$ of cases.

Potential risk factors for medication errors in patients with $\mathrm{eGFR} \leq \mathbf{4 0}$ alerts

Compared to the subjects without medication errors $(\mathrm{n}=1157)$, subjects for whom medication adjustments were recommended $(\mathrm{n}=211)$ were more often female subjects $(59 \%$ vs $41 \%, \mathrm{p}=0.04)$ and had a lower eGFR (median $34(28,38)$ versus $29(2,34) \mathrm{ml} / \mathrm{min} / 1.73 \mathrm{~m}^{2}$, $\mathrm{p}<0.001$, respectively). Notably, the latter had higher rates of polypharmacy $(70 \%$ vs $89 \%, \mathrm{p}<0.001$, mean number of medications $6.6(3.8)$ vs $8.2(3.5), \mathrm{p}<0.001)$.

\section{Effectiveness: pADEs and occurrence of ADEs after} follow-up

Overall, $88 \%(\mathrm{n}=299)$ of the medication errors were regarded as relevant pADEs (score $>0$ ). These were mainly judged to be either significant or serious. An overview of the number and potential severity of pADEs in the study population is given in figure 1 .

Overall, 40 ADEs were identified in hospital records within 1 year after the study period in the group of
Figure 4 Type of medication errors identified by the pharmacists.

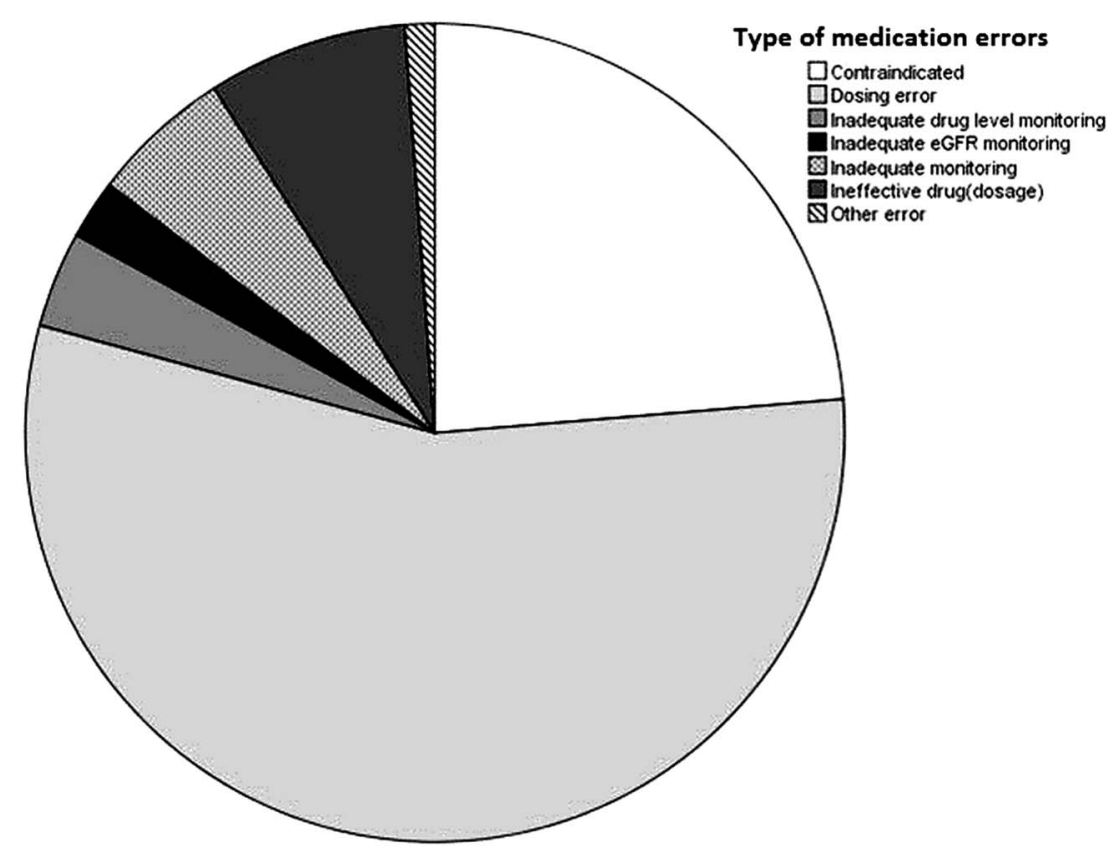


Figure 5 Type and frequency of recommended medication adjustments by community pharmacists.

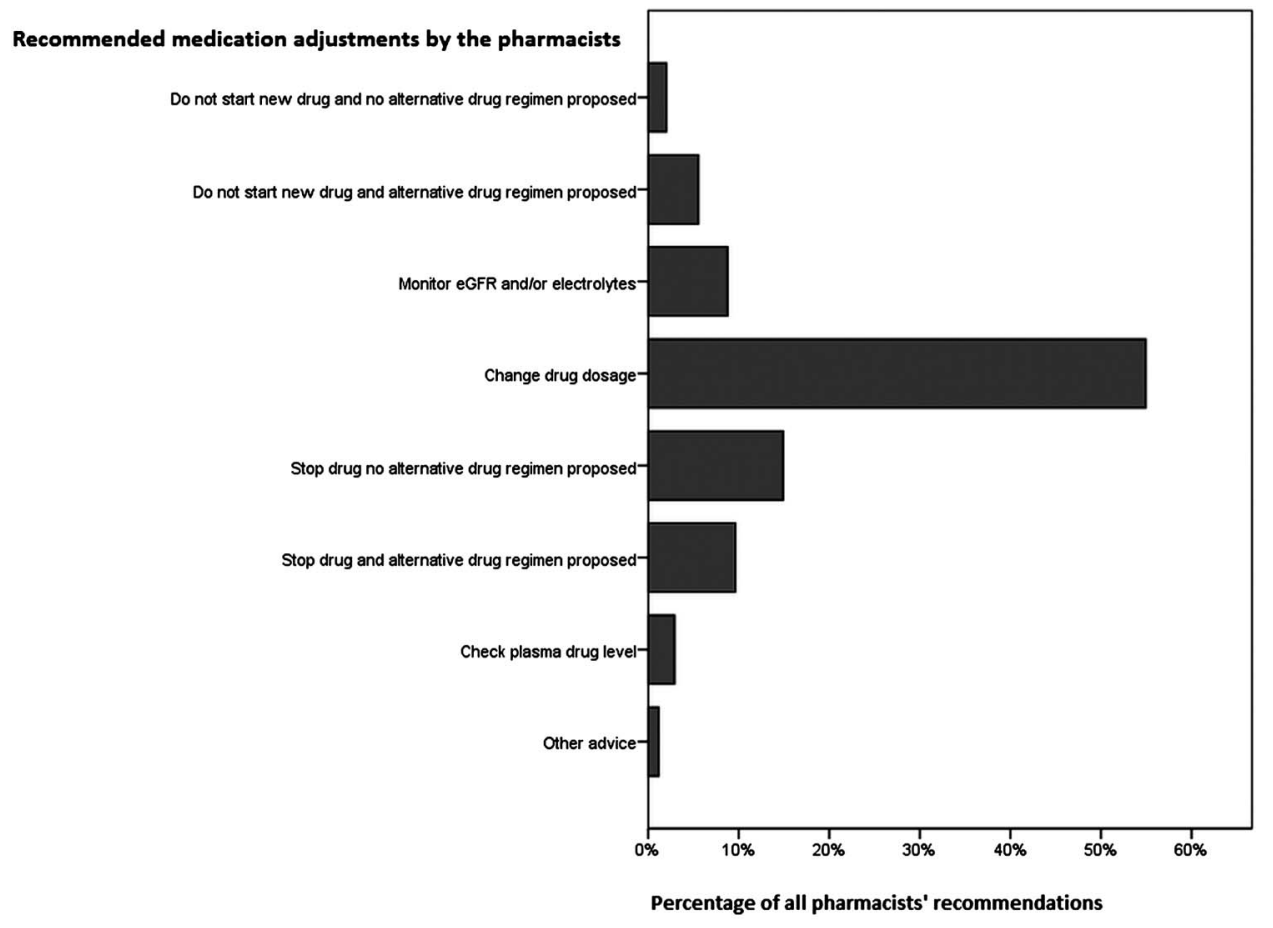

subjects with medication errors, including two lifethreatening ADEs (bradycardia due to digoxin intoxication and acute kidney injury with lactic acidosis associated with persistent metformin use). The number and severity of ADEs are shown in figure 1. Importantly, the $\mathrm{ADE}$ risk was higher in subjects whose medication regimen remained unchanged $(n=60)$ as compared to subjects whose medication regimen was adjusted as recommended by the pharmacist $(n=139) ; 38 \%$ vs $6 \%$, respectively.

\section{Effectiveness: workload and time investment of the pharmacists}

After receiving an eGFR $\leq 40$-alert, the pharmacist needed an average of 11 mins (range 5-13 mins) to check an individual's medication regimen for errors. When taking into account the time needed for consultation with the prescribing physician, pharmacists required an average of $20 \mathrm{mins}$ to process one eGFR $\leq 40$-alert triggering a medication adjustment. All pharmacists judged the time investment as feasible, particularly considering the fact that each pharmacy received an average of only one alert per week. Retrospectively, we evaluated the feasibility of different thresholds for kidney function alerts by calculating the number of low eGFR-alerts that would have been generated during the study period using different cut-offs for renal impairment $\left(<30,<50\right.$ and $<60 \mathrm{ml} / \mathrm{min} / 1.73 \mathrm{~m}^{2}$, respectively, see online supplementary appendix $\mathrm{B})$.

Overall, 904 eGFR $\leq 40$-alerts were activated in the records of the participating pharmacies at the end of the study period, as $16 \%(n=214)$ of the population died and in 250 subjects, the most recent eGFR was at least twice $>50 \mathrm{ml} / \mathrm{min} / 1.73 \mathrm{~m}^{2}$. Therefore, on average, every primary care pharmacy had 82 patients with an activated eGFR $\leq 40$-alert. If we translate this to a standard Dutch GP practice $( \pm 2300$ patients $)$, simple laboratory data sharing identified approximately 23 patients per practice who need drug adjustment (s) or extra alertness in medication management.

\section{DISCUSSION}

The main findings of this study were that an eGFR $\leq 40$-alert was indicated in $5.3 \%$ of the adult population of a Dutch city in whom a creatine measurement was performed in an ambulatory setting and that in these subjects 342 medication errors (mainly involving antibiotics and diuretics) were detected during the year following the introduction of an automatic eGFR $\leq 40$-alert system. The majority of the medication errors were regarded as relevant pADEs, necessitating medication Physicians complied in $66 \%$ of cases. ADE risk increases with age, polypharmacy and in instances where the proposed medication adjustments were initially rejected. Overall, automatically generated low eGFR-alerts in primary care seemed effective, easy to implement, and, importantly, improve both the pharmacists' and the physicians' awareness of medication safety.

\section{Comparison with other studies}

Despite the fact that medications are usually both prescribed and dispensed in the primary care setting, most studies on ( p)ADEs have been hospital based. ${ }^{39} 10$ We aimed to study the incidence of (p)ADEs in a shared pharmaceutical care model with a central role for community pharmacists. Three primary healthcare studies adjustments as recommended by the pharmacists. 
on this topic reported lower pharmacist drug proposal rates $(0.7-1.9 \%)$ than the $15 \%$ we found. ${ }^{31-33}$ These studies were performed in a general population, while we selected a high-risk population of subjects with renal impairment. In line with our results, primary and ambulatory care studies evaluating pharmacists' drug proposals in vulnerable subgroups like the elderly or subjects with cardiovascular risk factors also reported higher rates. ${ }^{12} 173435$ Two recent studies, also concerning subjects with renal impairment, identified problems related to inappropriate prescribing in over $20 \%$ of patients. ${ }^{18} 36$

Patients with renal impairment are especially vulnerable to medication errors. ${ }^{12}{ }^{13}{ }^{18}$ Various strategies to improve drug safety in these patients have been studied, such as educational wards rounds, immediate clinicianpharmacist feedback or dose adjustment according to renal function at hospital discharge. ${ }^{12} 18$ 37-42 However, despite the fact that most prescribing takes place in the primary healthcare setting, the majority of the strategies implemented so far have been tailored to hospital settings and are therefore not suitable for primary care. Others have demonstrated the effectiveness of 'computerised physician order entry' and 'clinical decision support' in reducing medication errors in case of renal impairment. ${ }^{39-41}$ However, computerised drug prescribing alerts do not always guarantee a reduction of prescribing errors, ${ }^{43}$ partly because such alerts are often overridden or ignored by prescribing physicians. ${ }^{41} 44-46$ This phenomenon is also reflected in our data, as in $28 \%$ of cases pharmacist recommendations were rejected by the prescribing physician.

A central role for community pharmacists in improving medication safety in primary care has been recognised. Many pharmacists are gradually extending their role as integral members of the medical team around the patient, thereby taking an important position in a shared care environment. ${ }^{21} 4748$ This has not only been induced by legislative issues, ${ }^{21}{ }^{25}$ but also recommended in various guidelines and studies to counteract problems associated with multiple medication prescribers. ${ }^{20} 263248$ This is important in view of our ageing population in which complex drug therapy will only increase, polypharmacy is common and renal impairment widespread. ${ }^{49} 50 \mathrm{~A}$ recent review showed notable differences in ADE prevalence rates by age groups, increasing from $5 \%$ for adults up to $16 \%$ for the elderly. ${ }^{7}$ Therefore, in complex cases (as with renal impairment), close collaboration between community pharmacists and physicians is essential to prevent ADEs.

The alert method we have investigated here could be a simple solution to address this.

Our strategy included three steps to reduce medication errors in patients with renal impairment. First, automatic laboratory alerts were generated; second, these alerts were linked to pharmacy data to judge the need for drug adjustments; and third, pharmacists discussed the recommended changes with physicians. Several studies investigated the impact of the above steps. The introduction of automatically generated laboratory alerts had varied effects on the prescribing physician. ${ }^{41} 51 \quad 52$ Authors suggested that such passive alerts did not have enough of an impact. There are limited data on the effect of extending the alerts so that the community pharmacist was also involved. Other studies showed that when the pharmacy data were linked with the laboratory renal data, the medication dosage could be beneficially adjusted. ${ }^{12} 4253$ We aimed to optimise medication safety in cases of renal impairment by combining the aforementioned steps and tailored our strategy for application in the primary care setting.

\section{Implications for clinical practice}

The estimated prevalence of both moderate $(30-59 \mathrm{ml} /$ $\left.\min / 1.73 \mathrm{~m}^{2}\right)$ and severe $\left(15-29 \mathrm{ml} / \mathrm{min} / 1.73 \mathrm{~m}^{2}\right)$ renal insufficiency in the adult American and Dutch population is $4.5 \%$ and $5.3 \%$, respectively. ${ }^{26}{ }^{54}$ Therefore, the number of subjects potentially susceptible to related medication errors is substantial. If we compile our pADE-rate towards nationwide figures (based on 12500000 adults in the Netherlands), our type of data sharing could intercept more than 40000 potential ADEs related to renal impairment each year. This would undoubtedly increase healthcare safety with already available data and (hopefully) decrease the costs of ADE-related morbidities. Drug safety management might be further improved by extending patient data exchange towards other important parameters, such as medication allergies, platelet counts, electrolyte concentrations, international normalised ratio, liver enzymes and plasma drug levels.

\section{Strengths and weaknesses of the study}

Some limitations of this study have to be noted. First, our study design does not allow the determination of either the individual healthcare effects or the overall cost-benefits. This would necessitate a more complex study design as was, for example, used in the populationbased randomised controlled renal drug alert effectiveness trial of Bhardwaja $e t a l^{36}$ or a 'before and after' design. However, participating GPs and pharmacists indicated that the protocol improved their awareness of medication errors related to renal function impairment. Second, data on the incidence of ADEs before the start of the study project were not available in our region; therefore, a possible change in ADE incidence as a result of our interventions cannot be determined. Besides, the incidence of ADEs is most likely underestimated due to underreporting, missed recognition and lack of recording in daily clinical practice. Our study also has several strengths. First, our intervention can be easily implemented in various healthcare settings. We simply extended the availability of laboratory renal data which were not shared formerly. Second, physicians valued the pharmacists' involvement in improving healthcare delivery. The acceptance percentage of the pharmacists was fairly good $(67 \%)$, as compared to previous studies (24-82\%), ${ }^{1732343751}$ and our prescription 
ratio between GPs and hospital-based physicians (77: 23\%) reflects the normal distribution of prescriptions in the Netherlands $(82: 18 \%) .^{55}$ However, to improve the overall efficiency of the eGFR-alerts, variables influencing physicians' (non) adherence to pharmacists' recommendations (like type and duration of medication use) should also be further studied. Third, the time investment was acceptable and costs were low. Finally, we chose a safe but also feasible threshold for renal function alerts. However, as thresholds for dosage adjustment vary between different guidelines, a higher cut-off of $\leq 50$ or $60 \mathrm{ml} / \mathrm{min} / 1.73 \mathrm{~m}^{2}$ or drug-specific thresholds could be discussed. ${ }^{25} \quad 26 \quad 36 \quad 56$ Besides, as the Cockcroft-Gault (CG) formula is often used in pharmacokinetic studies and for drug dosing recommendations, the implications of the use of renal function estimates, like the MDRD equations for drug dosing, are under debate. Several studies have compared drug dosing recommendations based on the CG with those based on the MDRD.$^{57-59}$ In summary, the accuracy of the MDRD seems comparable to the CG. ${ }^{57-59}$ On the basis of these studies, in our opinion, the MDRD is a reasonable alternative to the CG for drug dosing. This is of importance, especially since there is an increasing trend of clinical laboratories reporting the MDRD along with serum creatine, which is also recommended by national and international organisations. ${ }^{26}{ }^{60}$ Some guidelines advise a higher cut-off point for dose adjustments (creatine clearance $50-60 \mathrm{ml} / \mathrm{min}$ ), ${ }^{11}{ }^{61}$ but this was expected to result in an amount of alerts exceeding an acceptable workload. Moreover, as the MDRD tends to underestimate true GFR, we presumably already included subjects with true GFR $>40 \mathrm{ml} / \mathrm{min}^{62}$

\section{Conclusions and policy implications}

The introduction of automatic renal function alerts in the ambulatory care setting, with the involvement of both GPs and community pharmacists, revealed that a considerable part of the population is at risk for ADEs due to impaired renal function. Extending the availability of renal laboratory data to community pharmacists resulted in their presenting the prescribing physicians with a considerable number of medication adjustment recommendations. We feel that nationwide implementation of this simple protocol could potentially identify many pADEs and substantially reduce the risks of iatrogenic damage in persons with decreased renal function.

\footnotetext{
Author affiliations

${ }^{1}$ University of Groningen, University Medical Centre Groningen, Department of Internal Medicine, Groningen, The Netherlands

${ }^{2}$ Isala Clinics, Department of Internal Medicine, Diabetes Centre, Zwolle, The Netherlands

${ }^{3}$ Pharmacy De Fenix (previously Boogerd Kluin), Zwolle, The Netherlands ${ }^{4}$ Isala Clinics, Department of Clinical Chemistry and Laboratory Medicine, Zwolle, The Netherlands

${ }^{5}$ University of Groningen, University Medical Centre Groningen, Department of Internal Medicine, University Center for Geriatric Medicine, Groningen, The Netherlands
}

${ }^{6}$ GP Practice de Fenix, Zwolle, The Netherlands

${ }^{7}$ GP Practice Assendorp, Zwolle, The Netherlands

Acknowledgements We would like to thank the community pharmacists, the dispensary employees and the general practitioners in Zwolle for their participation in this study. We would also like to acknowledge Anton Huisman (laboratory staff) and Ellen Witteveen (medical student), who both took care of data collection, and Marcel Ariës (neurologist) for his careful comments on the manuscript. We thank Anne Starreveld for assistance on amending the text of the manuscript.

Contributors KJB, EVvdP, RJS, TJJ, OS and HJGB conceived the idea of the study and were responsible for the design of the study. HJ and ID were responsible for undertaking the data analysis and produced the tables and graphs. KJB, EVvdP and RJS provided input into the data analysis. The initial draft of the manuscript was prepared by HJ and HJGB and then circulated repeatedly among all authors for critical revision. $\mathrm{HJ}$ was responsible for the acquisition of the data and JPJS, ROBG, KJB, EVvdP, ID and HJGB contributed to the interpretation of the results. All authors had full access to all of the data (including statistical reports and tables) in the study and can take responsibility for the integrity of the data and accuracy of the data analysis.

Funding This research received no specific grant from any funding agency in the public, commercial or not-for-profit sectors.

Competing interests None.

Provenance and peer review Not commissioned; externally peer reviewed.

Data sharing statement No additional data are available.

\section{REFERENCES}

1. Leendertse AJ, Egberts AC, Stoker LJ, et al. HARM Study Group. Frequency of and risk factors for preventable medication-related hospital admissions in the Netherlands. Arch Intern Med 2008;168:1890-6.

2. Patel P, Zed PJ. Drug-related visits to the emergency department: how big is the problem? Pharmacotherapy 2002;22:915-23.

3. Beijer HJ, De Blaey CJ. Hospitalisations caused by adverse drug reactions (ADR): a meta-analysis of observational studies. Pharm World Sci 2002;24:46-54.

4. Gandhi TK, Weingart SN, Borus J, et al. Adverse drug events in ambulatory care. N Engl J Med 2003;348:1556-64.

5. Gurwitz JH, Field TS, Harrold LR, et al. Incidence and preventability of adverse drug events among older persons in the ambulatory setting. JAMA 2003;289:1107-16.

6. Thomsen LA, Winterstein AG, Søndergaard B, et al. Systematic review of the incidence and characteristics of preventable adverse drug events in ambulatory care. Ann Pharmacother 2007;41:1411-26.

7. Taché SV, Sönnichsen A, Ashcroft DM. Prevalence of adverse drug events in ambulatory care: a systematic review. Ann Pharmacother 2011;45:977-89.

8. Guthrie B, McCowan C, Davey $\mathrm{P}$, et al. High risk prescribing in primary care patients particularly vulnerable to adverse drug events: cross sectional population database analysis in Scottish general practice. BMJ 2011;342:d3514.

9. Bates DW, Cullen DJ, Laird N, et al. For the ADE Prevention Study Group. Incidence of adverse drug events and potential adverse drug events: implications for prevention. JAMA 1995;247:29-34.

10. Hug BL, Witkowski DJ, Sox CM, et al. Occurrence of adverse, often preventable, events in community hospitals involving nephrotoxic drugs or those excreted by the kidney. Kidney Int 2009;76:1192-8.

11. Munar MY, Singh $\mathrm{H}$. Drug dosing adjustments in patients with chronic kidney disease. Am Fam Physician 2007;75:1487-96.

12. Yap C, Dunham D, Thompson J, et al. Medication dosing errors for patients with renal insufficiency in ambulatory care. Jt Comm J Qual Patient Saf 2005;31:514-21.

13. Minutolo R, De Nicola L, Mazzaglia G, et al. Detection and awareness of moderate to advanced CKD by primary care practitioners: a cross-sectional study from Italy. Am J Kidney Dis 2008;52:444-53.

14. Fink JC, Brown J, Hsu VD, et al. CKD as an underrecognized threat to patient safety. Am J Kidney Dis 2009;53:681-8.

15. Stevens LA, Fares G, Fleming J, et al. Low rates of testing and diagnostic codes usage in a commercial clinical laboratory: evidence 
for lack of physician awareness of chronic kidney disease. J Am Soc Nephrol 2005;16:2439-88.

16. Hu KT, Matayoshi A, Stevenson FT. Calculation of the estimated creatinine clearance in avoiding drug dosing errors in the older patient. Am J Med Sci 2001;322:133-6.

17. Patel HR, Prunchnicki MC, Hall LE. Assessment for chronic kidney disease service in high-risk patients at community health clinics. Ann Pharmacother 2005;39:22-7.

18. Desrochers JF, Lemieux JP, Morin-Bélanger C, et al. Development and validation of the PAIR (Pharmacotherapy Assessement In chronic Renal disease) criteria to assess medication safety and use issues in patients with CKD. Am J Kidney Dis 2011;58:527-35.

19. Franke L, Avery AJ, Groom L, et al. is there a role for computerized decision support for drug dosing in general practice? A questionnaire survey. J Clin Pharm Ther 2000;25:373-7.

20. Kuo GM, Buckley TE, Fitzsimmons DS, et al. Collaborative drug therapy management services and reimbursement in a family medicine clinic. Am J Health-Syst Pharm 2004;61:343-54.

21. Chen YF, Neil KE, Avery AJ, et al. Prescribing errors and other problems reported by community pharmacists. Ther Clin Risk Manag 2005;1:333-42.

22. Molokhia M, Curcin V, Majeed A. Improving pharmacovigilance. Use of routinely collected data. BMJ 2010;340:c2403.

23. Schiff GD, Klass D, Peterson J, et al. Linking laboratory and pharmacy: opportunities for reducing errors and improving care. Arch Intern Med 2003;163:893-900.

24. Centraal Bureau voor de Statistiek (CBS). http://www.cbs.nl. (access date 1 Feb 2011)

25. Koninklijke Nederlandse Maatschappij ter bevordering der Pharmacie (KNMP). Verminderde nierfunctie; doseringsadviezen voor geneesmiddelenDen Haag: Geneesmiddel Informatie Centrum Editie 2009. ISBN 978-90-812445-4-1.

26. De Grauw WJC, Kaasjager HAH, et al. Landelijke transmurale afspraak (LTA) chronische nierschade. Huisarts Wet 2009;52:586-97.

27. Levey AS, Coresh J, Greene T, et al. Chronic Kidney Disease Epidemiology Collaboration. Expressing the Modification of Diet in Renal Disease Study equation for estimating glomerular filtration rate with standardized serum creatinine values. Clin Chem 2007:53:766-72.

28. Anatomical Therapeutic Chemical (ATC) classification system. http:// www.whocc.no/atc. Entry date 1 Feb 2009 (accessed 26 Feb 2009)

29. Morimoto T, Gandhi TK, Seger AC, et al. Adverse drug events and medication errors; detection and classification methods. Qual Saf Health Care 2004;13:306-14.

30. Egbring M, Far E, Knuth A, et al. Performance of different data sources in identifying adverse drug events in hospitalized patients. Eur J Clin Pharmacol 2011;67:909-18.

31. Rupp MT, DeYoung M, Schondelmeyer SW. Prescribing problems and pharmacist interventions in community practice. Med Care 1992;30:926-40.

32. Hawksworth GM, Corlett AJ, Wright DJ, et al. Clinical pharmacy interventions by community pharmacists during the dispensing process. Br J Clin Pharmacol 1999;47:695-700.

33. Benrimoj SI, Langford $\mathrm{JH}$, Berry G, et al. Clinical intervention rates in community pharmacy; a randomized trial of the effect of education and a professional allowance. Int J Pharm Pract 2003;11:71-80.

34. Monane M, Matthias DM, Nagle BA, et al. Improving prescribing patterns for the elderly through an online drug utilization review intervention: a system linking the physician, pharmacist, and computer. JAMA 1998;280:1249-52.

35. Zermansky AG, Petty DR, Raynor DK, et al. Randomised controlled trial of clinical medication review by a pharmacist of elderly patients receiving repeat prescriptions in general practice. $B M J$ 2001;323:1340-3

36. Bhardwaja B, Caroll NM, Raebel MA, et al. Improving prescribing safety in patients with renal insufficiency in the ambulatory setting: The Drug Renal Alert Pharmacy (DRAP) Program. Pharmacotherapy 2011;31:346-56.

37. Nash IS, Rojas M, Hebert $\mathrm{P}$, et al. Reducing excessive medication administration in hospitalized adults with renal dysfunction. $A m \mathrm{~J}$ Med Qual 2005;20:64-9.

38. Falconnier AD, Haefeli WE, Schoenenberger RA, et al. Drug dosage in patients with renal failure optimized by immediate concurrent feedback. J Gen Intern Med 2001;16:369-75.
39. Goldberg DE, Baardsgaard G, Johnson MT, et al. Computer-based program for identifying medication orders requiring dosage modification based on renal function. Am J Hosp Pharm 1991:48:1965-9.

40. Chertow GM, Lee J, Kuperman GJ, , et al Guided medication dosing for inpatients with renal insufficiency. JAMA 2001;286:2839-44.

41. McCoy AB, Waitman LR, Gadd Cs, et al. A computerized provider order entry intervention for medication safety during acute kidney injury; a quality improvement report. Am J Kidney Dis 2010;56:832-41.

42. Van Dijk EA, Drabbe NR, Kruijtbosch M, et al. Drug dosage adjustments according to renal function at hospital discharge. Ann Pharmacother 2006;40:1254-60.

43. Gandhi TK, Weingart SN, Seger AC, et al. Outpatient prescribing errors and the impact of computerized prescribing. $J$ Gen Intern Med 2005;20:837-41.

44. Isaac T, Weissman JS, Davis RB, et al. Overrides of medication alerts in ambulatory care. Arch Intern Med 2009;169:305-11.

45. Weingart SN, Toth M, Sands DZ, et al. Physicians' decisions to override computerized drug alerts in primary care. Arch Intern Med 2003;163:2625-31.

46. Galanter WL, Moja J, Lambert BL. Using computerized povider order entry and clinical decision support to improve prescribing in patients with decreased GFR. Am J Kidney Dis 2010;56:809-12.

47. Warholak TL, Rupp MT. Analysis of community chain pharmacists' interventions on electronic prescriptions. J Am Pharm Assoc 2003:49:59-64.

48. Bell JS, Rosen A, Aslani P, et al. Developing the role of pharmacists as members of community mental health teams: perspectives of pharmacists and mental health professionals. Res Social Adm Pharm 2007;3:392-409.

49. Olvaei AJ, Bennett WM. Drug dosing in the elderly patients with chronic kidney disease. Clin Geriatr Med 2009;25:459-27.

50. Wetzels JF, Kiemeney LA, Swinkels DW, et al. Age- and gender-specific reference values of estimated GFRin Caucasians: the Nijmegen Biomedical Study. Kidney Int 2007;72:632-7.

51. Tegeder I, Levy M, Muth-Selbach U, et al. Retrospective analysis of the frequency and recognition of adverse drug reactions by means of automatically recorded laboratory signals. Br J Clin Pharmacol 1999:47:557-64.

52. Sellier E, Colombet I, Sabatier B, et al. Effect of alerts for drug dosage adjustment in inpatients with renal insufficiency. J Am Med Inform Assoc 2009;16:203-10.

53. Schiff GD, Klass D, Peterson J, et al. Linking laboratory and pharmacy: opportunities for reducing errors and improving care. Arch Intern Med 2003;28:893-900.

54. Coresh J, Astor BC, Greene T, et al. Prevalence of chronic kidney disease and decreased kidney function in the adult US population: Third National Health and Nutrition Examination Survey. Am J Kidney Dis 2003;41:1-12.

55. Pharmaceutisch Weekblad 2008:38:13. http://www.pw.nl/archief/ 2008/2008-38/2008pw38p13.pdf/view (accessed 4 Dec 2011)

56. Long CL, Raebel MA, Price DW, et al. Compliance with dosing guidelines in patients with chronic kidney disease. Ann Pharmacother 2004;38:853-8.

57. Stevens LA, Nolin TD, Richardson MM, et al. Comparison of drug dosing recommendations based on measured GFR and kidney function estimating equations. Am J Kidney Dis 2009;54:33-42.

58. De Lemos ML, Hsieh T, Hamata L et al. Evaluation of predictive formulae for glomerular filtration rate for carboplatin dosing in gynaecological malignancies. Gynecol Oncol 2006;103:1063-69.

59. Stevens LA, Levey AS. Use of the MDRD study equation to estimate kidney function for drug dosing. Clin Pharmacol Ther 2009;86:465-7.

60. National Kidney Foundation. K/DOQI clinical practice guidelines for CKD evaluation, classification, and stratification. Am J Kidney Dis 2002;39:S1-266.

61. Hanlon JT, Aspinall SL, Semla TP, et al. Consensus guidelines for oral dosing of primarily renally cleared medications in older adults. $J$ Am Geriatr Soc 2009:57:335-40.

62. Stevens LA, Coresh J, Greene T, et al. Assessing kidney functionmeasured and estimated glomerular filtration rate. $N$ Engl $J$ Med 2006;354:2473-83 